\section{Ultrasonographic findings of posterior interosseous nerve syndrome}

\author{
Youdong Kim, Doo Hoe Ha, Sang Min Lee \\ Department of Radiology, CHA Bundang Medical Center, CHA University, Seongnam, Korea
}

Purpose: The purpose of this study was to evaluate the ultrasonographic findings associated with posterior interosseous nerve (PIN) syndrome.

Methods: Approval from the Institutional Review Board was obtained. A retrospective review of 908 patients' sonographic images of the upper extremity from January 2001 to October 2010 revealed 10 patients suspicious for a PIN abnormality (7 male and 3 female patients; mean age of $51.8 \pm 13.1$ years; age range, 32 to 79 years). The ultrasonographic findings of PIN syndrome, including changes in the PIN and adjacent secondary changes, were evaluated. The anteroposterior diameter of the pathologic PIN was measured in eight patients and the anteroposterior diameter of the contralateral asymptomatic PIN was measured in six patients, all at the level immediately proximal to the proximal supinator border. The size of the pathologic nerves and contralateral asymptomatic nerves was compared using the Mann-Whitney $U$ test.

Results: Swelling of the PIN proximal to the supinator canal by compression at the arcade of Fröhse was observed in four cases. Swelling of the PIN distal to the supinator canal was observed in one case. Loss of the perineural fat plane in the supinator canal was observed in one case. Four soft tissue masses were noted. Secondary denervation atrophy of the supinator and extensor muscles was observed in two cases. The mean anteroposterior diameter of the pathologic nerves $(n=8,1.79 \pm 0.43 \mathrm{~mm}$ ) was significantly larger than that of the contralateral asymptomatic nerves $(n=6,1.02 \pm 0.22 \mathrm{~mm})(P=0.003)$.

Conclusion: Ultrasonography provides high-resolution images of the PIN and helps to diagnose PIN syndrome through visualization of its various causes and adjacent secondary changes.

Keywords: Radial nerve; Posterior interosseous nerve; Nerve compression syndromes; Ultrasonography

\section{Introduction}

The posterior interosseous nerve (PIN) is the deep motor branch of the radial nerve. The radial nerve, after coursing anterior to the lateral epicondyle of the humerus, bifurcates into a superficial sensory branch and the PIN at the level of the radiocapitellar joint. After coursing through the radial tunnel, the PIN passes between the superficial and deep heads of the supinator muscle. After exiting the supinator canal, the PIN supplies the extensor compartment muscles of the forearm [1].

Nerve entrapment syndrome is a neuropathy caused by structural abnormalities. It may be caused by extrinsic compression, displacement, or traction of the nerve due to masses, anomalous muscles,

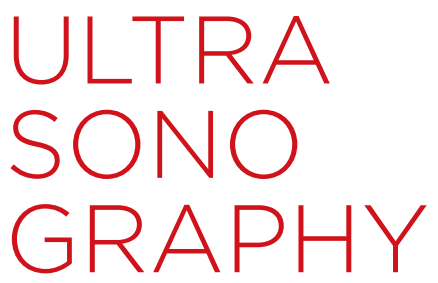

ORIGINAL ARTICLE

https://doi.org/10.14366/usg. 17007 pISSN: 2288-5919 - elSSN: 2288-5943

Ultrasonography 2017:36:363-369

Received: February 2, 2017

Revised: April 2, 2017

Accepted: April 5, 2017

Correspondence to:

Doo Hoe Ha, MD, Department of Radiology, CHA Bundang Medical Center, CHA University, 59 Yatap-ro, Bundang-gu, Seongnam 13496, Korea

Tel. +82-31-780-5424

Fax. +82-31-780-5381

E-mail:dhha@cha.ac.kr

This is an Open Access article distributed under the terms of the Creative Commons Attribution NonCommercial License (http://creativecommons.org/ licenses/by-nc/3.0/) which permits unrestricted noncommercial use, distribution, and reproduction in any medium, provided the original work is properly cited.

Copyright (C) 2017 Korean Society of Ultrasound in Medicine (KSUM)

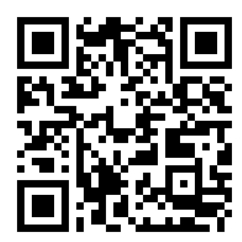

How to cite this article:

Kim Y, Ha DH, Lee SM. Ultrasonographic findings of posterior interosseous nerve syndrome. Ultrasonography. 2017 Oct;36(4): 363-369. 
fibrous bands, or osseous deformities. Predisposing factors include overuse, trauma, and long-term external compression [2-4]. It can also be caused by an intrinsic abnormality of the nerve itself, such as a neurogenic tumor $[5,6]$. PIN entrapment syndrome, or simply PIN syndrome, is a rare entity compared to other well-known compressive neuropathies such as carpal tunnel syndrome and cubital tunnel syndrome. The annual incidence of PIN syndrome is $0.003 \%$ [7], and it accounts for less than $0.7 \%$ of all upper limb peripheral nerve syndromes [8]. The arcade of Fröhse, the tendinous proximal edge of the supinator muscle, is the most common cause of PIN syndrome. Other structures, including the borders of adjacent muscles and vessels, can also compress the PIN [1]. Ganglion cysts, lipoma, or synovial pathologies, as well as radial head fracture and Monteggia fracture-dislocation, may also cause compression of the PIN $[9,10]$. The clinical findings of PIN syndrome include weakness in the extension of the fingers, atrophy of the forearm muscles, and localized pain in the lateral aspect of the elbow and proximal forearm [1].

Nerve entrapment syndromes are usually diagnosed through a nerve conduction study or needle electromyography (EMG) [2]. EMG has the advantage of localizing the point of neuropathy and establishing the electrophysiologic severity of the lesion [11]. However, it may be insufficient for a thorough evaluation due to the lack of concrete spatial information. Imaging studies can identify the primary cause of entrapment. Of the various imaging modalities, ultrasonography has become widely accepted as the initial imaging modality for peripheral nerves [2].

However, only a limited number of case reports $[12,13]$ and case series describing the sonographic features of PIN syndrome are available in the literature. Moreover, previous studies focused on the arcade of Fröhse, but not on other lesions that result in entrapment and secondary changes in the nearby structures. Hence, the purpose of this study was to evaluate the ultrasonographic findings of PIN syndrome, with a particular focus on its various causes and adjacent secondary changes.

\section{Materials and Methods}

Approval from the Institutional Review Board was obtained and informed consent was waived. From January 2001 to October 2010, 980 patients were referred to our Department for Ultrasonography of the upper extremity, from below the shoulder joint through the wrist joint. The patients were referred for ultrasonography for various indications, such as weakness, mass, pain, or sensory changes. The PIN was most commonly examined in patients with weakness in the extension of the fingers or pain in the forearm. The review identified 12 patients with pathologic findings of the PIN. Ultrasonography of two patients without symptoms attributable to entrapment of the PIN were excluded, and 10 patients were included in this study.

Seven patients were men and three patients were women. The mean age of patients was $51.8 \pm 13.1$ years, ranging from 32 to 79 years. The right arm was involved in eight cases and the left arm in two cases. The chief complaint of nine patients was weakness of the fingers or metacarpophalangeal joints, and for the remaining one patient the chief complaint was a mass in the forearm and numbness of the ipsilateral thumb and index finger. The mean duration of symptoms was $8.9 \pm 8.3$ weeks, ranging from 1 week to 6 months. Of the nine patients who complained of weakness, there were predisposing factors in six patients; trauma $(n=2)$, overuse of the forearm $(n=2)$, and long-term compression by sleeping on the forearm $(n=2)$.

EMG was performed in nine patients and revealed a PIN injury in seven patients. No evidence of PIN injury was noted in two patients (ulnar neuropathy, $n=1$; no peripheral neuropathy, $n=1$ ). In three patients (EMG not performed, $n=1$; EMG performed but negative for PIN injury, $n=2$ ), PIN syndrome was diagnosed by surgical removal of the mass and the subsequent improvement of symptoms.

Ultrasonography was performed by one of two experienced musculoskeletal radiologists, with more than 10 and 15 years of experience, respectively, using linear array transducers (8-15 $\mathrm{MHz}$ for Acuson Sequoia, Siemens Medical Solutions, Erlangen, Germany and 5-12 MHz for IU22, Philips, Amsterdam, Netherlands). Ultrasonography was performed with the patient seated or lying down and with the arm in extension. The arm was initially in supination when examining the radial tunnel, and gradually shifted to pronation as the transducer was moved distally to trace the PIN in the supinator muscle.

Ultrasonographic findings were reviewed by the both of the musculoskeletal radiologists who performed the ultrasonography examinations, and they reached a consensus for each examination. The ultrasonographic findings of PIN syndrome were evaluated for any change in the PIN, including swelling, compression, displacement, changed echogenicity, and the presence of a mass originating from or in the vicinity of the PIN. Adjacent secondary changes, such as loss of the perineural fat plane and denervation atrophy of the supinator and extensor muscles, were also evaluated. If a mass was not identified or if the radiologist was not convinced of the effect of a mass on the pathologic PIN, the contralateral asymptomatic PIN was evaluated, and the ultrasonographic findings were compared.

Swelling of the nerve was defined as an increase in diameter with hypoechoic changes and an indistinct fascicular morphology of the PIN compared to its more proximal level or the same level of the contralateral asymptomatic nerve. Compression of the nerve 
was defined as a decrease in diameter of the PIN, compared to its adjacent levels or the same level of the contralateral asymptomatic nerve, due to an external structure. Displacement of the nerve was defined as an alteration in the normal anatomic course of the PIN. Denervation atrophy of a muscle was defined as diffuse hyperechoic or mixed hyperechoic and hypoechoic changes of a muscle originally innervated by the PIN. Perineural fat loss was defined as nonvisualization of the thin hyperechoic layer circumferentially surrounding the PIN.

The anteroposterior diameter was measured at the level immediately proximal to the proximal supinator border. The anteroposterior diameter was not measured for a pathologic PIN associated with neurogenic tumor $(n=1)$ since it could reflect the tumor size rather than the extent of swelling. Additionally, the anteroposterior diameter was not measured for swelling distal to the supinator $(n=1)$. Thus, the anteroposterior diameter of the pathologic PIN was measured in eight patients, and that of the contralateral asymptomatic PIN was measured in six patients.

The statistical analysis for comparison of the anteroposterior diameter of the eight pathologic PINs with the six contralateral

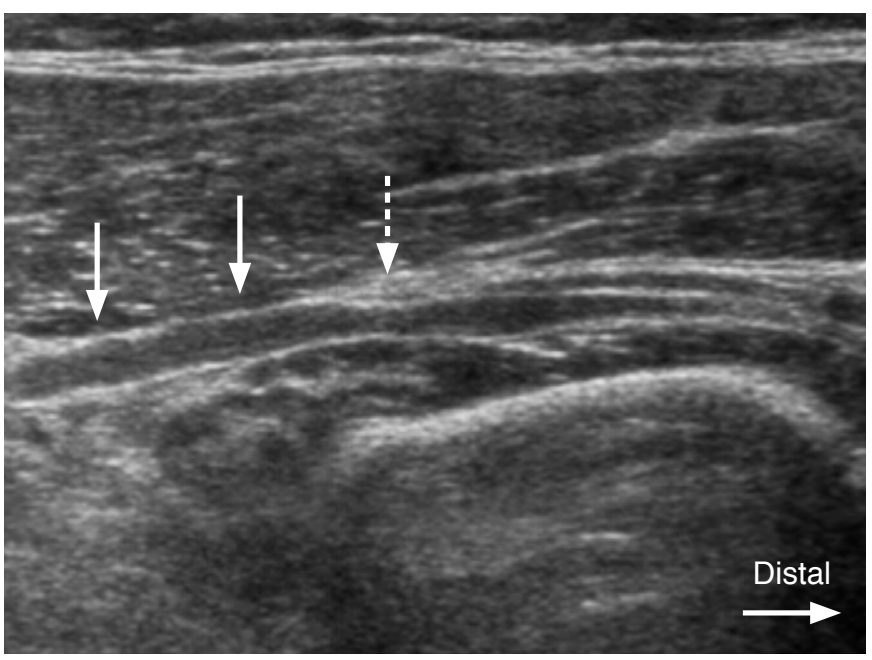

A

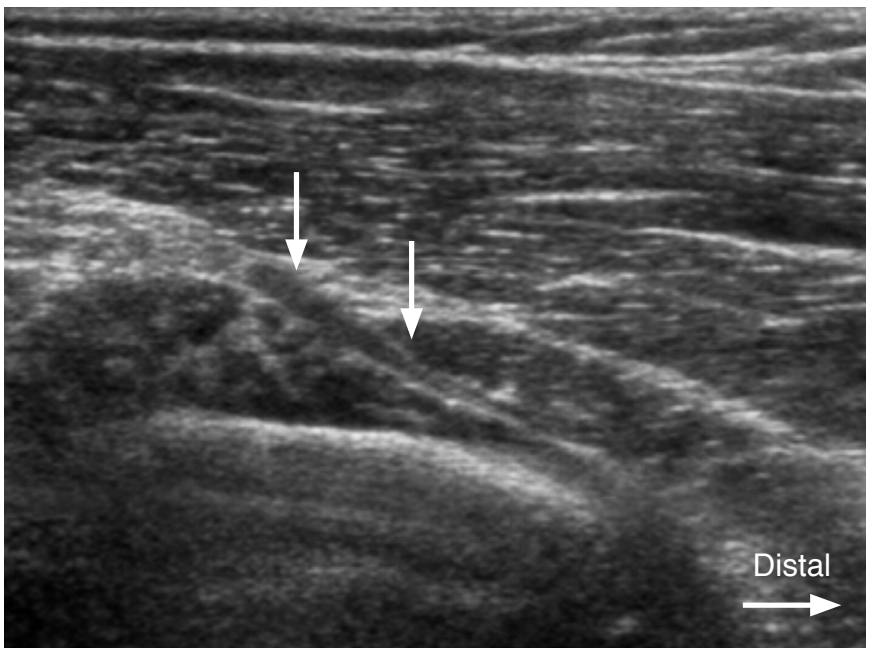

C

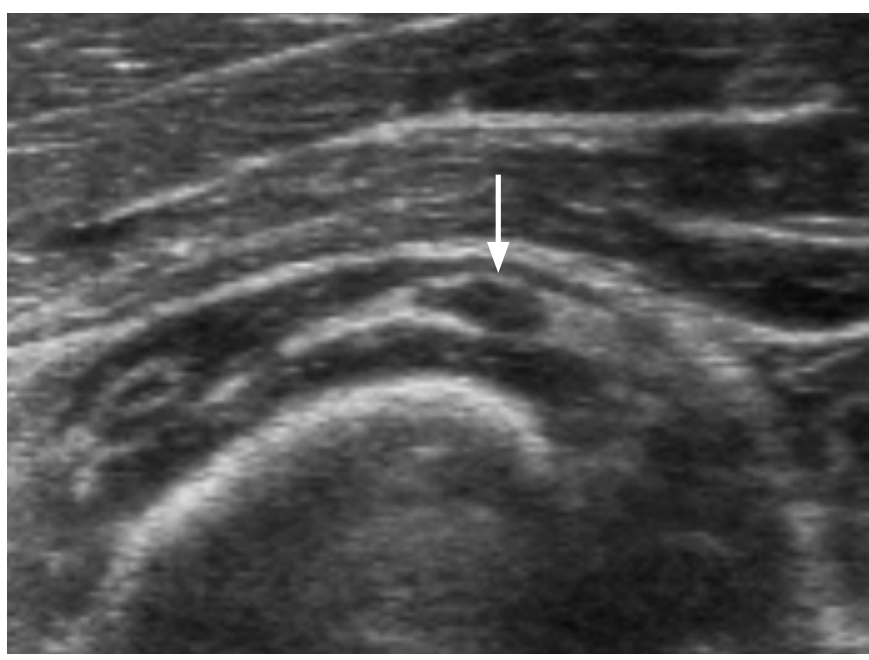

B

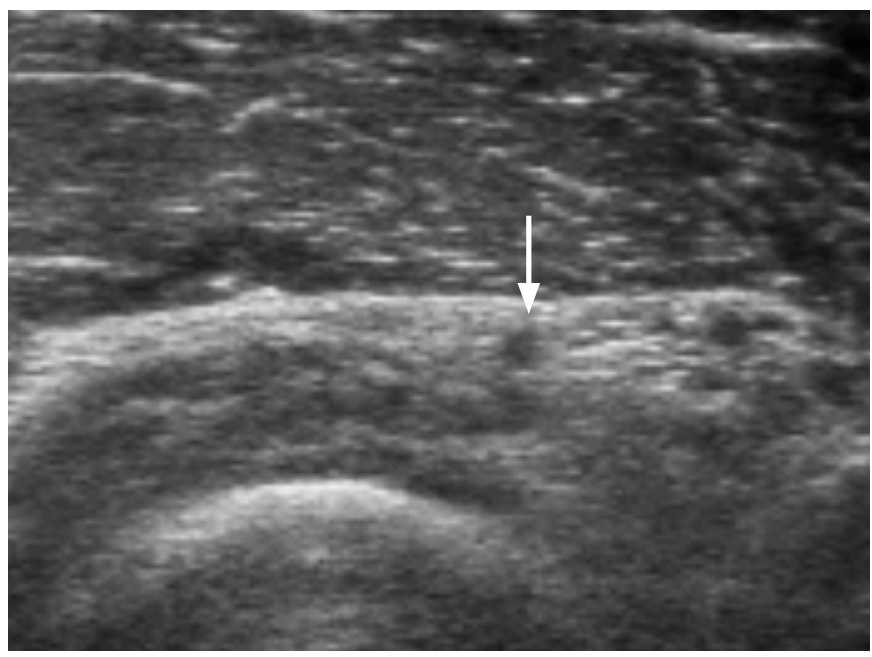

D

Fig. 1. A 42-year-old man with swelling of the posterior interosseous nerve (PIN) proximal to the supinator muscle.

A. Longitudinal ultrasonogrphy demonstrates hypoechoic swelling of the left PIN (arrows) proximal to the supinator muscle. Compression by the hyperechoic thickened arcade of Fröhse (dashed arrow) is noted. B. Axial ultrasonography shows swelling of the left PIN (arrow) at a level just proximal to the arcade of Fröhse. The thickness of the swollen PIN was measured as $2.4 \mathrm{~mm}$ in anteroposterior diameter. C, D. Ultrasonography of the contralateral asymptomatic right forearm shows a gradual tapering appearance of the PIN (arrows) in the longitudinal scan (C) with a normal thickness (1.0 mm in anteroposterior diameter) of the PIN (arrow) in an axial scan (D). 
asymptomatic PINs was performed with the Mann-Whitney U test using SPSS ver. 22 (IBM Corp., Armonk, NY, USA).

\section{Results}

Compression and proximal hypoechoic swelling of the PIN at the entrance into the supinator canal (the arcade of Fröhse) was observed in four of 10 cases (Figs. 1, 2). Swelling of the PIN immediately distal to the exit from the supinator was observed in

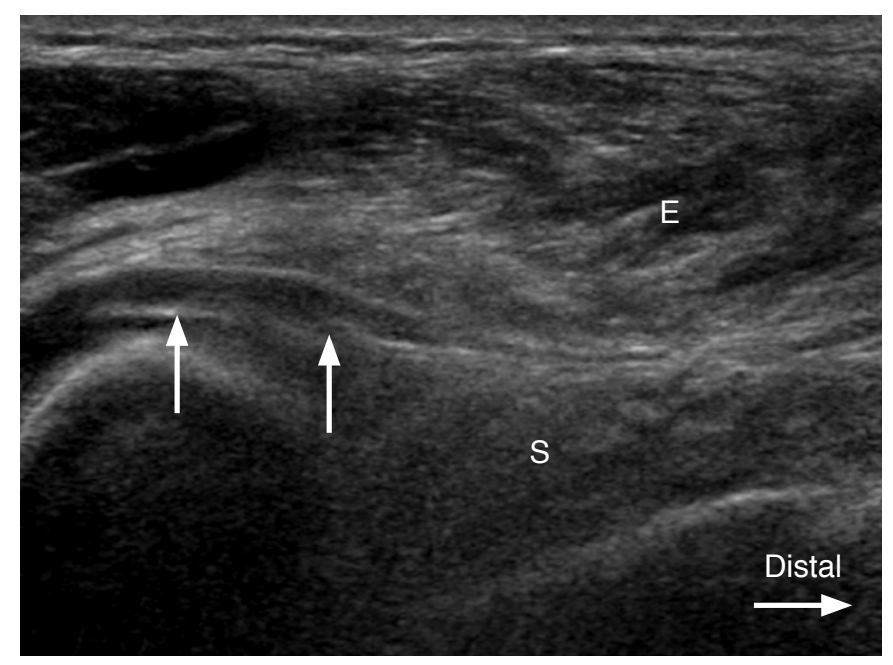

A one of the 10 cases (Fig. 3).

Four soft tissue masses ( 4 of 10 ) were noted. A ganglion cyst was noted in three of the 10 cases, of which two caused compression of the PIN, and the remaining ganglion cyst caused displacement of the PIN (Fig. 4). All three ganglion cysts were associated with swelling of the ipsilateral PIN, and a subsequent histologic confirmation was made by surgical resection. A neurogenic tumor of the PIN (1 of 10) was noted in the supinator canal in one case, and was surgically confirmed as myxoid neurofibroma.

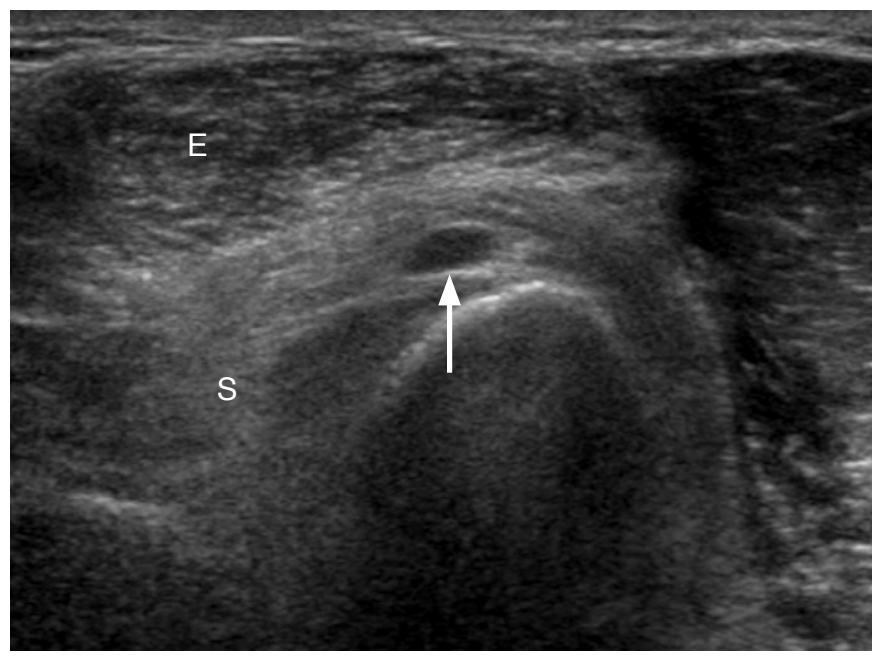

B

Fig. 2. A 51-year-old man with swelling of the posterior interosseous nerve (PIN) proximal to the supinator muscle. Longitudinal (A) and axial (B) ultrasonography demonstrates hypoechoic swelling of the PIN (arrows) just proximal to the entrance site into the supinator canal, and diffuse hyperechoic change of the supinator (S) and mixed hyperechoic and hypoechoic changes of the extensor (E) muscles, which represent denervation atrophy.

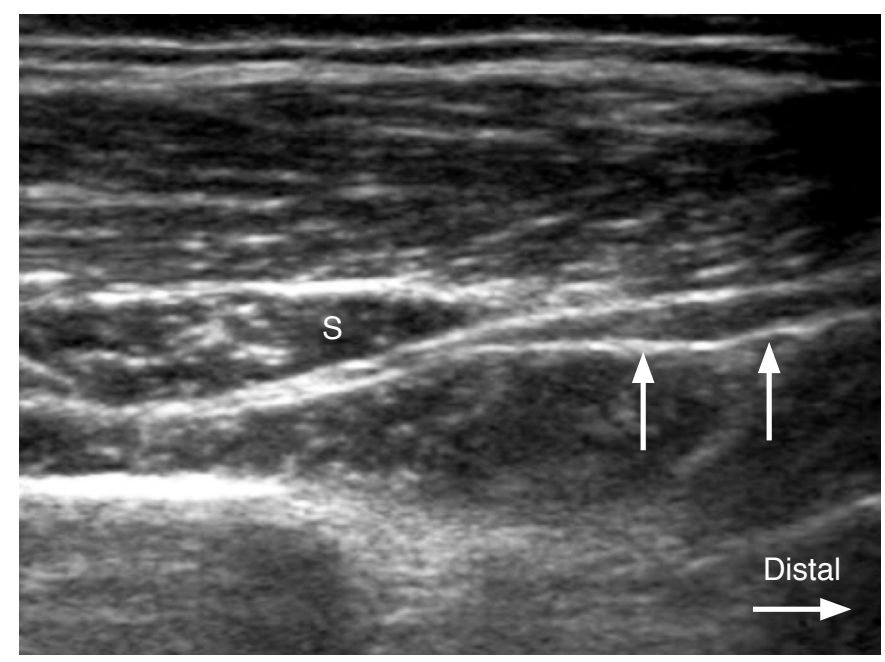

A

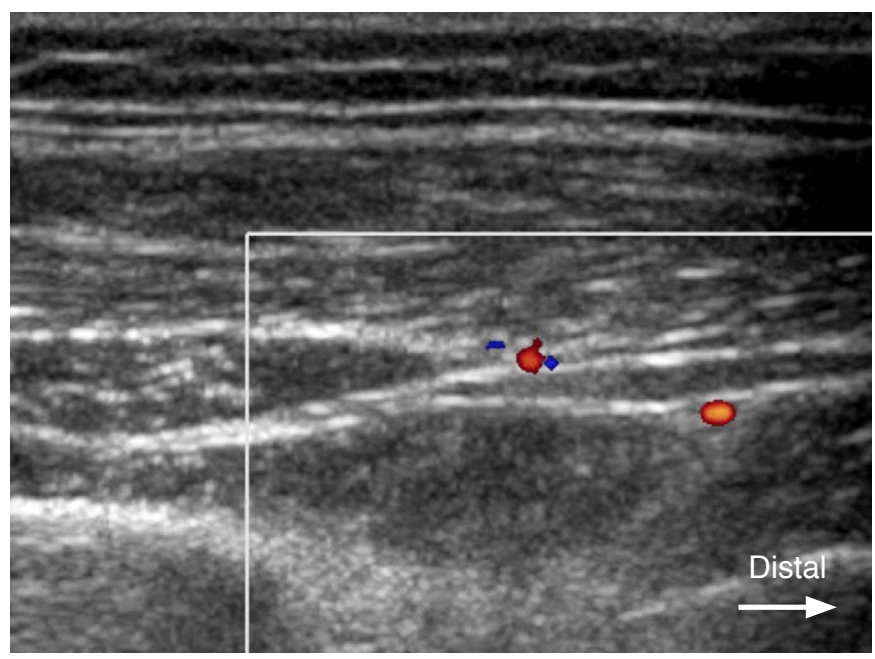

B

Fig. 3. A 49-year-old woman with swelling of the posterior interosseous nerve (PIN) distal to the supinator muscle.

A. Gray-scale ultrasonography shows hypoechoic swelling of the PIN (arrows) as it exits from the supinator canal (S). B. Color Doppler image demonstrates increased perineural vascularity at the site of hypoechoic swelling of the PIN. 


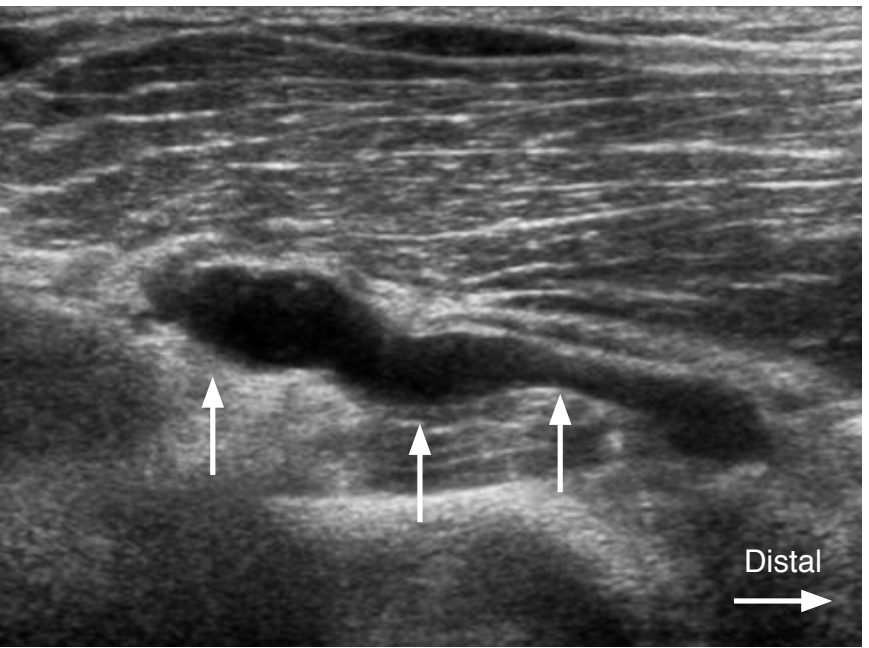

A

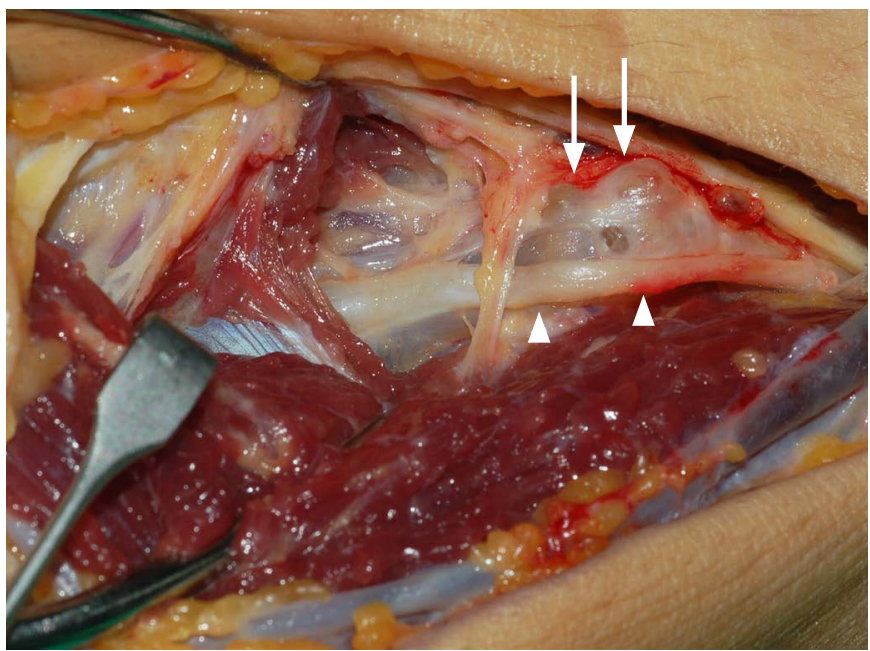

C

Loss of the perineural fat plane in the supinator canal with a normal-looking nerve was also observed in one case.

Denervation atrophy of the supinator, extensor digitorum communis, and extensor carpi ulnaris muscles was observed in two cases: in one case of PIN compression at the arcade of Fröhse (Fig. 2), and in the case of the neurogenic tumor.

No abnormal findings were noted in the seven contralateral asymptomatic PINs.

The mean anteroposterior diameter was $1.79 \pm 0.43 \mathrm{~mm}$ for the pathologic PINs, and $1.02 \pm 0.22 \mathrm{~mm}$ for the mean contralateral asymptomatic PINs. The size difference was statistically significant $(\mathrm{P}=0.003)$.

\section{Discussion}

PIN syndrome can be caused by extrinsic effects, such as

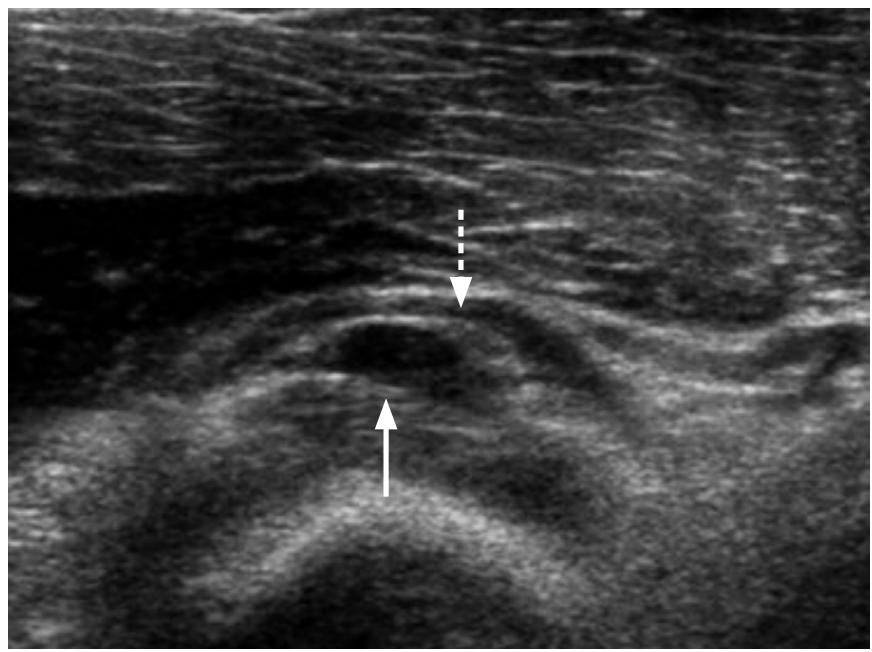

B

Fig. 4. A 54-year-old man with a ganglion cyst in the supinator canal.

A. A tubular, lobulated, anechoic ganglion cyst (arrows) is demonstrated from the site just proximal to the supinator muscle to the proximal supinator canal in a longitudinal ultrasonography. B. The posterior interosseous nerve (PIN) (dashed arrow) is displaced and followed due to an anechoic ganglion cyst (arrow) in the entrance site to the supinator canal in an axial ultrasonography. C. Surgical photograph demonstrates a ganglion cyst (arrows) and displaced PIN (arrowheads).

compression on the PIN from adjacent structures, or by intrinsic lesions of the nerve. Five points of potential compression of the PIN have been identified. The most proximal point is the fibrous band of the radial head, which is continuous with the extensor carpi radialis brevis, and the superficial head of the supinator. The next point, at the level of the radial neck, is the radial recurrent vessels, referred to as the leash of Henry. The third potential point is the tendinous margin of the extensor carpi radialis brevis. The fourth point is the aponeurotic proximal margin of supinator (the arcade of Fröhse). The last and most distal point is the distal margin of the supinator $[3,14]$.

The arcade of Fröhse has been reported to be the most common site of compression of the PIN [1]. It is the proximal edge of the superficial head of the supinator muscle. The arcade of Fröhse can be classified as tendinous, musculotendinous, muscular, or membranous. The tendinous type is considered a significant risk factor for PIN syndrome, with the incidence ranging from $64 \%$ 
to $87 \%$ in different studies [10]. In this study, of the six patients without masses (a ganglion cyst or a neurogenic tumor), four patients showed swelling immediately proximal to the level of the arcade of Fröhse.

Compression by the arcade of Fröhse is aggravated by repetitive pronation and supination of the forearm [15]. In supination, the PIN moves laterally, lengthens, and rotates in the anticlockwise direction as the superficial layer of the supinator muscle is relaxed. In pronation, the opposite situation occurs, in which the superficial layer of the supinator muscle is tightened and compresses the PIN passively [10].

The mechanism by which the arcade of Fröhse and other adjacent structures such as the thickened superomedial border of the extensor carpi radialis or the leash of Henry cause symptoms in PIN syndrome is compression-induced neuronal swelling. Pathophysiologically, the response of the nerve to compression is endoneural inflammation, edema, fibrosis, demyelination, and remyelination. This process eventually results in thickening of the endoneurium and perineurium, leading to ischemia and obstruction of normal cytoplasmic axonal transport, which is termed axonal damming [16].

To date, only two clinical trials on the ultrasonography of PIN syndrome have been published in the literature, to the best of our knowledge. Bodner et al. [17] evaluated four patients with PIN syndrome and 10 healthy volunteers via ultrasonography. The diameters of the PINs of the patients $(3.3 \mathrm{~mm})$ were greater than those of the volunteers $(1.3 \mathrm{~mm})$, with unknown statistical significance. Djurdjevic et al. [6] examined 13 patients with PIN syndrome caused only by the compression at the arcade of Fröhse and 20 volunteers. The mean anteroposterior PIN diameter was $1.6 \mathrm{~mm}$ for patients and $1.1 \mathrm{~mm}$ for volunteers. This difference between the two groups was statistically significant. In the present study, the mean anteroposterior diameter was $1.79 \pm 0.43 \mathrm{~mm}$ for the pathologic nerves and $1.02 \pm 0.22 \mathrm{~mm}$ for the contralateral asymptomatic nerves, with statistical significance.

Both of the aforementioned studies examined PINs that were compressed by the arcade of Fröhse. In this study, of the 10 patients examined, only four cases were attributable to compression by the arcade of Fröhse, whereas four cases were caused by ganglion cysts and neurogenic tumors, showing diverse etiologies compared to the previous studies of the ultrasonographic findings associated with PIN syndrome. In addition to the diverse etiologies, this study revealed ultrasonographic findings other than swollen hypoechoic nerves. In one case, loss of the perineural fat plane in the supinator canal was the only pathologic finding of the PIN itself, and decreased echogenicity of the supinator was observed. In two cases, denervation atrophy of the supinator, extensor carpi radialis brevis, extensor digitorum communis, and extensor carpi ulnaris muscles was observed. In contrast to hypoechoic swelling of the PIN proximal to the supinator muscles in cases of compression by the arcade of Fröhse, swelling distal to the supinator muscle was also observed in one case without any other abnormal findings.

Isolated neuritis of the PIN has been reported in the literature as a cause of PIN syndrome [18]. Nakamichi and Tachibana [18] reported hourglass-like constriction of the nerve with an intraneural hyperechoic band at the level of the capitellum. In our study, one patient showed nerve swelling distal to the supinator muscle without apparent compression by any anatomic structure in the vicinity, raising the possibility of isolated neuritis.

Another useful tool other than ultrasonography for diagnosis of neuropathy is EMG. The usefulness of ultrasonography in relation to EMG has not been clearly elucidated. In a study by Mondelli et al. [19], 70 patients clinically suspected to have mononeuropathy of the radial nerve or the PIN underwent EMG studies alone, without ultrasonography. EMG evidence of radial nerve or PIN injury was observed in 61 patients $(87 \%)$, whereas nine patients (13\%) did not show any EMG abnormality. In the study by Djurdjevic et al. [6], only patients who had abnormal EMG findings were sent for ultrosonography, and all 13 patients referred for ultrasonography showed edematous change of the PIN. In our study, EMG was performed in nine cases. Seven cases (78\%) had a PIN injury, one case revealed no abnormal findings, and one case showed ulnar neuropathy. In the case with normal EMG findings, ultrasonography revealed a neurogenic tumor of the PIN with denervation injury of the extensor muscles, and subsequent surgery confirmed the diagnosis. In the case where EMG indicated ulnar neuropathy, ultrasonography demonstrated a ganglion displacing the PIN, and this was also subsequently confirmed by surgery.

It is known that abnormal EMG activity may be missed in cases studied earlier than 3 weeks or later than 12 weeks after the onset of neuropathy [19]. Both of the cases above with no indication of PIN injury fell into this time period. Thus, ultrasonography can play an essential role in diagnosing PIN syndrome in particular and possibly other upper arm neuropathies in general, especially in cases where the duration between the onset of symptoms and the performance of an imaging or EMG study is shorter than 3 weeks or longer than 12 weeks.

Another advantage of ultrasonography is direct high-resolution visualization of the PIN itself throughout the entire supinator canal and adjacent anatomic structures. This enables the examiner to identify various potential causative lesions, as well as secondary denervation atrophy of the affected muscles.

The main limitation of this study lies in its retrospective nature, which could have biased the results. Additionally, the small sample size of 10 patients could have led to biased results as 
well. Even though the size difference between the pathologic nerves and contralateral asymptomatic nerves was statistically significant, a larger sample size would be desirable. The lack of EMG in some patients, and the different time periods in relation to ultrasonography in those who did undergo EMG, are other limitations. Moreover, the findings were not confirmed by surgical pathology in all patients. Finally, this study lacked asymptomatic volunteers for comparison.

In conclusion, ultrasonography helps in the diagnosis of PIN syndrome through visualization of its various causes and adjacent secondary changes.

ORCID: Youdong Kim: http://orcid.org/0000-0003-4778-508X; Doo Hoe Ha: http:// orcid.org/0000-0003-0744-5697

\section{Conflict of Interest}

No potential conflict of interest relevant to this article was reported.

\section{References}

1. Martinoli C, Bianchi S, Pugliese F, Bacigalupo L, Gauglio C, Valle M, et al. Sonography of entrapment neuropathies in the upper limb (wrist excluded). J Clin Ultrasound 2004;32:438-450.

2. Roquelaure Y, Raimbeau G, Dano C, Martin YH, Pelier-Cady $M C$, Mechali $S$, et al. Occupational risk factors for radial tunnel syndrome in industrial workers. Scand J Work Environ Health 2000;26:507-513.

3. Konjengbam M, Elangbam J. Radial nerve in the radial tunnel: anatomic sites of entrapment neuropathy. Clin Anat 2004;17:2125.

4. Ebnezar J. Textbook of orthopedics. 4th ed. New Delhi: Jaypee Brothers Medical Publishers Ltd., 2010.

5. Miller TT, Reinus WR. Nerve entrapment syndromes of the elbow, forearm, and wrist. AJR Am J Roentgenol 2010;195:585-594.

6. Djurdjevic T, Loizides A, Loscher W, Gruber H, Plaikner M, Peer S. High resolution ultrasound in posterior interosseous nerve syndrome. Muscle Nerve 2014;49:35-39.

7. Latinovic R, Gulliford MC, Hughes RA. Incidence of common compressive neuropathies in primary care. J Neurol Neurosurg Psychiatry 2006;77:263-265.

8. Hazani R, Engineer NJ, Mowlavi A, Neumeister M, Lee WP, Wilhelmi BJ. Anatomic landmarks for the radial tunnel. Eplasty 2008;8:e37.

9. Yamazaki H, Kato H, Hata Y, Murakami N, Saitoh S. The two locations of ganglions causing radial nerve palsy. J Hand Surg Eur Vol 2007;32:341-345.

10. Portilla Molina AE, Bour C, Oberlin C, Nzeusseu A, Vanwijck R. The posterior interosseous nerve and the radial tunnel syndrome: an anatomical study. Int Orthop 1998;22:102-106.

11. Fernandez E, Pallini R, Lauretti L, Scogna A, Di Rienzo A. Neurosurgery of the peripheral nervous system: the posterior interosseous nerve syndrome. Surg Neurol 1998;49:637-639.

12. Chien AJ, Jamadar DA, Jacobson JA, Hayes CW, Louis DS. Sonography and MR imaging of posterior interosseous nerve syndrome with surgical correlation. AJR Am J Roentgenol 2003;181:219-221.

13. Kinni V, Craig J, van Holsbeeck M, Ditmars D. Entrapment of the posterior interosseous nerve at the arcade of Frohse with sonographic, magnetic resonance imaging, and intraoperative confirmation. J Ultrasound Med 2009;28:807-812.

14. Thomas SJ, Yakin DE, Parry BR, Lubahn JD. The anatomical relationship between the posterior interosseous nerve and the supinator muscle. J Hand Surg Am 2000;25:936-941.

15. Clavert $P$, Lutz JC, Adam P, Wolfram-Gabel R, Liverneaux P, Kahn JL. Frohse's arcade is not the exclusive compression site of the radial nerve in its tunnel. Orthop Traumatol Surg Res 2009;95:114-118.

16. Weiss P. Endoneurial edema in constricted nerve. Anat Rec 1943;86:491-522.

17. Bodner G, Harpf C, Meirer R, Gardetto A, Kovacs P, Gruber H. Ultrasonographic appearance of supinator syndrome. J Ultrasound Med 2002;21:1289-1293.

18. Nakamichi K, Tachibana S. Ultrasonographic findings in isolated neuritis of the posterior interosseous nerve: comparison with normal findings. J Ultrasound Med 2007;26:683-687.

19. Mondelli M, Morana P, Ballerini M, Rossi S, Giannini F. Mononeuropathies of the radial nerve: clinical and neurographic findings in 91 consecutive cases. J Electromyogr Kinesiol 2005;15:377-383. 